\title{
PHYSICAL PARAMETER MAPPING OF ACCRETION DISKS
}

\author{
S. VRIELMANN ${ }^{1}$, F. V. HESSMAN ${ }^{1}$, K. HORNE $^{2}$, \\ R. BAPTISTA ${ }^{2}$ \\ 1. Universitäts-Sternwarte Göttingen, Germany \\ 2. University of St. Andrews, Scotland, UK
}

Abstract. We present a further development of the classical eclipse mapping method, which reconstructs the spatial distribution of physical parameters in the accretion disks of cataclysmic variables.

\section{Limitations of classical eclipse mapping}

The classical 'eclipse-mapping' method (Horne 1985; Baptista \& Steiner 1991) employs a maximum-entropy approach (MEM) (Skilling \& Bryan 1984) to derive the spatial distribution of the continuum or line intensities of accretion disks in cataclysmic variables. This widely used method has revealed circular disks with more or less pronounced hot spots and radially decreasing brightness temperature distributions (e.g. Wood, Horne \& Vennes 1992; Baptista et al. 1995).

The classical method, however, suffers from the following limitations: (i) a single data set is mapped into a single quantity (intensity); (ii) light curves at different wavelengths are assumed to be independent; (iii) the requirement of phase independence is contradicted by anisotropic emission, e.g. from the hot spot or from optically thick lines due to shear effects and turbulence (Horne \& Marsh 1986; Horne 1995) and (iv) the information in the line profiles (radial velocities) is ignored.

\section{A more physical approach}

We have extended the classical method by mapping physical parameters (e.g. temperature, density and height of the optically thin layer) instead of continuum or line intensities. Using data at several different wavelengths, one can use wisely chosen physical models to deduce a (hopefully) small number of physical parameter maps. Depending upon the success of the fit, different models with increasing complexity can be attempted: optically thick blackbody emission; optically thin LTE disk spectra; 'stellar 
atmosphere' spectra or the inclusion of NLTE effects. Furthermore, special effects can be easily included: anisotropic emission; velocity profiles; UV illumination by the central source.

\section{A simple test case}

As a first test of our method, we constructed $U B V$ light curves $(\mathrm{S} / \mathrm{N}=50)$ for a blackbody accretion disk. The simultaneous fits to the data are good (Fig. 1 left). The reconstructed temperature distribution (Fig. 1 right) qualitatively reproduces the general behaviour of the test distribution, but shows typical MEM effects: steep inner and outer gradients are smoothed and to compensate this, the smooth intermediate gradient is steepened. The large extension of the disk at temperatures of about $4000 \mathrm{~K}$ is also artificial: as the observed $U B V$ light curves are rather insensitive to regions as cold as $4000 \mathrm{~K}$ MEM produces a disk which is as flat as possible and still in agreement with the data. Since the effects of MEM are known, Physical Parameter Mapping can be considered as a promising tool to interpret the physical properties of accretion disks.
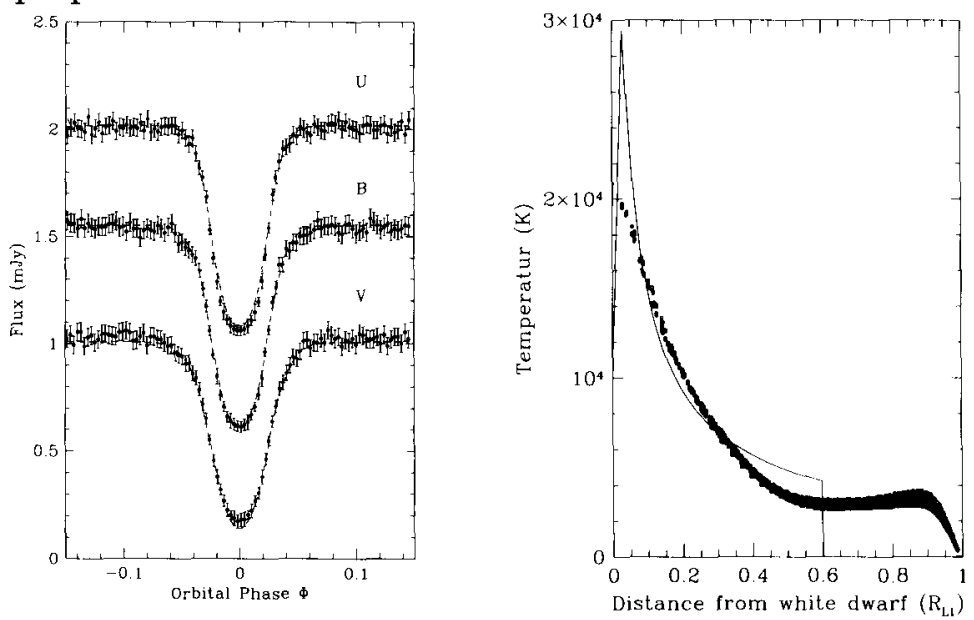

Figure 1. Left: Test $U B V$ eclipse light curves (dots with errorbars; $U, B$ shifted by $0.5 n \mathrm{mJy}$ ) and fitted eclipse light curves of the reconstructed disk image (dashed lines). Right: Temperature distributions of the test image (solid line) and of the reconstructed image (dots) derived by fitting all 3 light curves simultaneously.

\section{References}

Baptista, R., Steiner, J.E., 1991, A\&A, 249, 284

Baptista, R., Horne, K., Hilditch, R.W., et al., 1995, Ap. J., 448, 395

Horne, K., 1985, MNRAS, 213, 129

Horne, K., 1995, A\&A, 297, 273

Horne, K., Marsh, T.R., 1986, MNRAS, 218, 761

Skilling, J., Bryan, R.K., 1984, MNRAS, 211, 111

Wood, J.H., Horne, K., Vennes, S., 1992, Ap. J., 385, 294 